\title{
Markov Localization of Wireless Local Area Network Clients
}

\author{
Michael Wallbaum and Torsten Wasch* \\ Department of Computer Science, \\ RWTH Aachen University, D-52056 Aachen, Germany
}

\begin{abstract}
Markov localization has been successfully deployed in robotics using highly precise distance sensors to determine the location and pose of mobile robots. In this setting the scheme has shown to be robust and highly accurate. This paper shows how this approach has been adapted to the problem of locating wireless LAN clients in indoor environments using highly fluctuating radio signal strength measurements. A radio propagation model is used to determine the expected signal strength at a given position in order to avoid tedious offline measurements. Some of the issues that had to be addressed include expressing the calculated signal strengths in terms of probability density functions and detecting movement of the mobile terminal solely on the basis of radio measurements. The conducted experiments show that the proposed technique provides a median error of less than $2 \mathrm{~m}$ even when there is no line-of-sight to an access point.
\end{abstract}

\section{Introduction}

Geolocation systems in general can be based on a dedicated infrastructure or on an infrastructure designed for other purposes. The most popular approach to the latter is to use a wireless communication network. The spread of wireless LANs (WLAN) following the IEEE 802.11 standard [1] has consequently induced research on using the installed equipment for indoor geolocation purposes, as presented e.g. in 2, 3, 4] and 5. Without modifications to the hardware of commercially available WLAN products such location systems must rely on radio signal strength (RSS) measurements to determine a mobile terminal's (MT) position. The measured RSS is commonly compared to reference values of a socalled radio map determined in the offline phase, i.e. before MT's are actually localized.

The WLAN location determination scheme proposed in this paper is based on the concept of Markov localization by Dieter Fox et al. presented in [6]. The idea behind this approach is to use discrete probability distributions to represent the MT's state space. To avoid time-consuming reference measurements the system presented in this paper uses a propagation model to create a radio map. The Markov localization scheme proves to be sufficiently robust to compensate

* The work presented in this paper is supported by the German Federal Ministry of Education and Research under Grant 08NM211. 
for the errors introduced by the noisy wireless channel and by the propagation model. For real-world experiments its implementation has been integrated into the WhereMops location service introduced in [11.

The remainder of this paper is organized as follows. Section 2 describes the theoretical background to the proposed scheme, leaving some important - implementation specific - aspects open. These open issues will be tackled in Sect. 3 , which introduces a new probabilistic radio propagation model, and Sect. 4, which presents the implementation details of the location determination system. Section 5 describes the evaluation process and the obtained results. Section 6 concludes the paper and gives a preview of possible future work.

\section{Markov Localization}

\subsection{Motivation and Basic Idea}

In recent years probabilistic localization schemes such as Markov and MonteCarlo localization have gained increasing interest in the context of mobile robot systems designed for dynamic environments. Despite the fact that most mobile robots are equipped with rather precise distance sensor equipment deterministic approaches did not cope well in dynamic settings, e.g. with moving people or other objects not incorporated into the world model. Probabilistic approaches inherently model the uncertainties of real-world scenarios and can thus potentially achieve a higher robustness and accuracy.

The problem of dynamic environments also affects WLAN-based location determination systems, however such systems additionally suffer from highly inaccurate "distance sensors", meaning the measured received signal strength. Radio waves propagate in a very complex manner, the result being that even in a static environment one can observe so-called short term fading, i.e. fluctuations in the received signal strength. Furthermore even small displacements of the MT can significantly alter even the mean of the signal strength readings. The assumption at the outset of the work described in this paper, was that the Markov localization technique would prove sufficiently robust to cope with these adverse conditions, i.e. the dynamic office environment, the inaccurate sensor readings and the inaccurate world model provided by a propagation model, and yet deliver highly precise terminal locations.

Instead of maintaining a single hypothesis as to the MT's position, Markov localization maintains a probability distribution over the space of all such hypotheses. In the following this probability distribution is called belief. The belief about the MT's location is only updated when new perceptions are made (i.e. new RSS-measurements have been received) or after the MT has moved. The probabilistic representation allows the scheme to weigh these different hypotheses in a mathematically sound way.

\subsection{Notation}

Let $L_{T}$ denote the random variable representing the location of the MT at time $t=T$ and $l=(x, y)^{T} \in L$ a specific position of the MT within the state space. 
Following the notation used in [6] let $\operatorname{Bel}\left(L_{T}=l\right)$ denote the probability, that at time $t=T$ the MT is located at a position $l \in L$. In the following the continuous probability distribution is approximated by a discrete, grid-based representation. Positions $l$ that are located outside the grid have a probability of $\operatorname{Bel}\left(L_{T}=l\right)=0$. As $\operatorname{Bel}\left(L_{T}=l\right)$ represents a probability distribution the sum over all grid cell values is $\sum_{l \in L} \operatorname{Bel}\left(L_{T}=l\right)=1$.

Finally let $S_{T}=\left\langle s_{0}, s_{1}, \ldots, s_{T}\right\rangle$ denote the temporally ordered list of all RSS-measurements and $A_{T}=\left\langle a_{0}, a_{1}, \ldots, a_{T}\right\rangle$ the temporally ordered list of all movements conducted by the MT up to time $t=T$.

\subsection{Independence Assumptions}

This section describes two essential independence assumptions which allow for an efficient recursive implementation of the Markov-Localization algorithm.

Independence of Actions. The state $L_{T}$ at time $t=T$ solely depends on $L_{T-1}$ and the last conducted action - i.e. movement $-a_{T-1}$. In other words, all previously reached locations, all sensory input and all previously conducted actions become irrelevant once the current state $L_{T-1}$ is known. This is known as Markov-assumption and is summarized in the following equation:

$$
P\left(L_{T}=l \mid L_{T-1}, A_{T-1}, S_{T-1}\right)=P\left(L_{T}=l \mid L_{T-1}, a_{T-1}\right)
$$

Independence of Sensor Input. A sensor reading $s_{T}$ at time $t=T$ solely depends on the state of the environment at $t=T$. Once an MT's state space $L_{T}$ is known, all previously recorded measurements, states and actions provide no additional information for the calculation of $s_{t}$. Equation 2 summarizes this predication.

$$
P\left(s_{T} \mid L_{1}, \ldots, L_{T-1}, A_{T-1}, S_{T-1}\right)=P\left(s_{T} \mid L_{T}=l\right)
$$

\subsection{The Sensor Model}

The sensor model describes how to update the belief about an MT's position $l \in L$ at time $t=T$ given all previously recorded sensor readings $S_{T}$. This can be formulated as follows:

$$
P\left(L_{T}=l \mid S_{T}\right)=P\left(L_{T}=l \mid s_{0}, s_{1}, \ldots, s_{T}\right) .
$$

Using Bayes rule and Eq. 2 this can be transformed to

$$
=\frac{P\left(s_{T} \mid L_{T}=l\right) \cdot P\left(L_{T}=l \mid S_{T-1}\right)}{P\left(s_{T} \mid S_{T-1}\right)} .
$$

Obviously the denominator of Eq. 4 is independent of $L_{T}$ and therefore constant. Furthermore it is assumed that the probability $P\left(s_{T} \mid L_{T}=l\right)$ for a sensor reading given a certain position is time-invariant. Hence, using $\frac{1}{\alpha_{T}}=P\left(s_{T} \mid S_{T-1}\right)$ and the notation for $\operatorname{Bel}\left(L_{T}=l\right)$ introduced at the outset, Eq. 4 can be rewritten as:

$$
\operatorname{Bel}\left(L_{T}=l\right)=\alpha_{T} \cdot P\left(s_{T} \mid l\right) \cdot \operatorname{Bel}\left(L_{T-1}=l\right) .
$$


This states that the updated belief about the location of an MT upon new sensory input, depends on the probability of the sensory input at a given position weighted by the assumed likelihood of being at this position.

\subsection{The Action Model}

The belief about the MT's position is not only influenced by the current sensor readings, but also by actions (i.e. movements) of the terminal. Thus there is a need to calculate the probability $P(A)=P\left(L_{T}=l \mid A_{T-1}\right)$ that an MT at time $t=T$ is located at position $l \in L$, given all previously conducted movements $A_{T-1}$. Using the law of total probability this can be written as:

$$
P\left(L_{T}=l \mid A_{T-1}\right)=\sum_{l^{\prime}} P\left(A \mid B_{l^{\prime}}\right) \cdot P\left(B_{l^{\prime}}\right) .
$$

with $P\left(B_{l^{\prime}}\right)=P\left(L_{T-1}=l^{\prime} \mid A_{T-1}\right)$ and $P\left(A \mid B_{l^{\prime}}\right)=P\left(L_{T}=l \mid A_{T-1}\right.$, $\left.L_{T-1}=l^{\prime}\right)$.

Considering the assumption about independence of actions (Eq. 1) $P\left(A \mid B_{l^{\prime}}\right)$ can be simplified such that the probability is only dependent on the last conducted movement $a_{T-1}$. The term can be further simplified if it is assumed that the probability of reaching a location $l$ given a location $l^{\prime}$ and an action $a_{T-1}$ is time invariant.

$$
P\left(A \mid B_{l^{\prime}}\right)=P\left(l \mid a_{T-1}, L_{T-1}=l^{\prime}\right)
$$

Resubstituting Eq. 7 in Eq. 6] and using the definition of $\operatorname{Bel}\left(L_{T}=l\right)$ the influence of movements on the belief about the MT's location can thus be expressed as:

$$
\operatorname{Bel}\left(L_{T}=l\right)=\sum_{l^{\prime}} P\left(l \mid a_{T-1}, L_{T-1}=l^{\prime}\right) \cdot \operatorname{Bel}\left(L_{T-1}=l^{\prime}\right)
$$

This states that the probability of being at location $l$ after an action has been performed, can be calculated by summing up the probabilities of reaching $l$ from $l^{\prime}$ given action $a_{T-1}$. Each addend is weighted by the likelihood of starting at position $l^{\prime}$.

\subsection{Algorithm}

The previous section has presented the underlying principles of MarkovLocalization. Equation 5 provides a recursive scheme for updating the desired density $\operatorname{Bel}\left(L_{T}=l\right)$ when new sensor readings $s_{T}$ are available. Complementary to this Eq. 8 provides the recursive definition of the update procedure when movement of the MT has been detected. The complete algorithm in pseudo-code is shown in listing 2.1

So far four questions - all of which are highly dependent on the application environment - remain unanswered: 


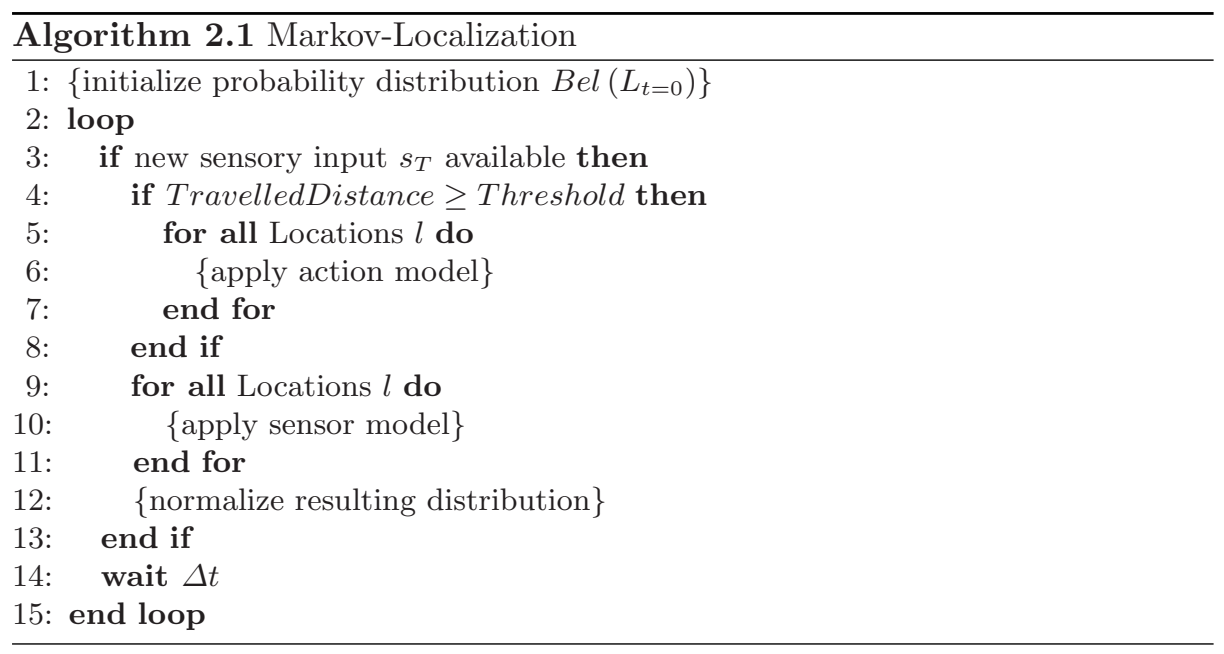

1. How is $P\left(s_{T} \mid l\right)$ (Eq. 5) calculated, i.e. the probability of sensor readings depending on the location?

2. How is $P\left(l \mid a_{T}, L_{T-1}=l^{\prime}\right)$ (Eq. 8) calculated, i.e. the probability of reaching one location from another given a movement?

3. How is movement detected based on RSS measurements?

4. How should the density function for $\operatorname{Bel}\left(L_{t=0}\right)$ be initialized?

All questions are answered in Sect. 4 which describes the implementation details of the Markov localizer. First however the automatic creation of radio maps is discussed, as this is an important prerequisite for the location determination system.

\section{Computing Radio Maps}

In order to calculate the likelihood of a signal strength measurement given a certain position and base station, the system needs to know what to expect. Radio (signal strength) maps are commonly used to associate reference positions with their expected radio signal strength. The simplest way to build a radio map is by conducting measurements for a set of reference points, with the obvious disadvantage being its enormous costs in terms of time. Trivially the positioning accuracy depends on the distance between the chosen reference points. For example, a desired positioning accuracy of $2 \mathrm{~m}$ on one floor of the computer science department building depicted in Fig. 1 would require approximately 300 measurements. Should the floor plan change or an access point be relocated these measurements would have to be repeated. In essence the empirical creation of radio maps is impractical especially considering large-scale deployment of location-based services.

A different approach to generating a radio map given a floor plan is to employ radio propagation models, which are frequently used to plan wireless communication networks. The advantage of calculating instead of measuring the radio 


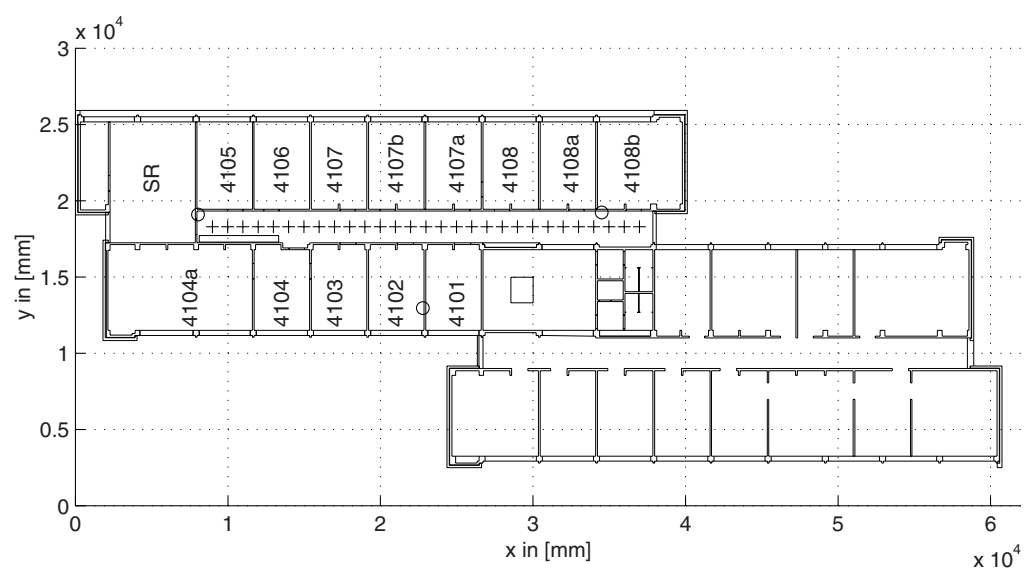

Fig. 1. Floor plan of Chair for Communication and Distributed Systems.

map is less effort and the allowance for arbitrarily fine grained grids. However, radio propagation models can only provide an estimate for the expected signal strengths as radio waves are subject to diverse and complex propagation phenomena. The following section briefly introduces the concept of empirical propagation models which are then modified for the purposes of generating radio maps for Markov localization.

\subsection{Empirical Propagation Models}

Empirical propagation models are based on the model presented in [8] which was adopted by Seidel und Rappaport to the conditions of indoor environments [9].

$$
n_{P}(d)=n_{P}\left(d_{0}\right)-10 \cdot \eta_{F S} \cdot \log _{10}\left(\frac{d}{d_{0}}\right)-x
$$

The first term on the right side of the equation describes the received signal strength at a reference distance $d_{0}$ from the sender under conditions of free-space propagation and is thus an indirect measure of the sender's transmitting power and antenna gain. The unit of $n_{P}$ is $\mathrm{dBm}$ and its value is usually determined by measurements. The second term models the free-space pathloss of the signal. The received signal strength decreases logarithmically with increasing receivertransmitter displacement $d$. The parameter $\eta_{F S}$ is called pathloss exponent and is also estimated by measurements. The last term models the variable attenuation of the signal due to obstructions in the environment.

The RADAR-system 2] employs such a model to calculate a radio map, whereby $X$ is made a function of the number of walls/obstacles between sender and receiver and an average attenuation for all obstacle types. This model is called Wall Attenuation Factor (WAF) model. 


\subsection{Design of a Probabilistic Propagation Model}

The modifications to the empirical propagation models mentioned above aimed at balancing the accuracy and the effort for estimating the model parameters, by classifying the different obstacle types in the environment. This achieves a higher accuracy than averaging the attenuation over all obstacles as in the WAF-model, yet it requires less effort than the FAF-model described in [10] which requires the attenuation for every specific obstacle.

The novelty of the proposed model is that it calculates an expected value for the RSS at a map location and a measure for the uncertainty or accuracy of the calculated value. These figures can be interpreted as mean and standard deviation of a normal distribution. The calculation of these two values is explained in the following.

Taking a classification of obstacles according to their attenuation properties, let $K$ denote the cardinality of the classification and $\eta_{k}^{\text {Wall }}$ the attenuation of the $k^{\text {th }}$ object class and $c_{k}$ the maximum number of objects of class $k$ to consider. Using the above definition the expected strength for a position at distance $d$ from an access point given a floor plan can be calculated as

$$
n_{P}(d)=n_{P}\left(d_{0}\right)-10 \cdot \eta_{F S} \cdot \log _{10}\left(\frac{d}{d_{0}}\right)-\sum_{k=1}^{K} \begin{cases}\mathcal{N}_{k} \cdot \eta_{k}^{W \text { all }} & \mathcal{N}_{k}<c_{k} \\ c_{k} \cdot \eta_{k}^{W \text { all }} & \mathcal{N}_{k} \geq c_{k}\end{cases}
$$

with

$$
\begin{aligned}
n_{P}\left(d_{0}\right) & : \text { signal strength at reference distance } d_{0} . \\
\eta_{F S} \geq 0 & : \text { free-space pathloss. } \\
\eta_{k}^{W a l l} \geq 0 & : \text { attenuation of object class } k . \\
\mathcal{N}_{k} & : \text { number of objects of class } k \text { between sender and receiver. } \\
c_{k} & : \text { maximum number of considered objects of class } k .
\end{aligned}
$$

Neither the WAF- nor the FAF-model attempt to estimate the deviation of the calculated expected signal strength to corresponding RSS-measurements. However the difference between model and reality is likely to increase with the distance and the number of obstacles between sender and receiver, as the corresponding parameters pathloss exponent and object attenuation can hardly be determined very precisely.

The uncertainty can be modelled as standard deviation of a probability distribution with mean as defined above by $n_{P}(d)$. To calculate the standard deviation we first define a helper function $\gamma(d) \forall d \geq 0$ which linearly maps the transmitter-receiver separation $d$ to the range $0 \leq \gamma(d) \leq 1$.

For evaluating the location determination scheme proposed in this paper the following two functions modelling the uncertainty have been developed and tested.

$$
\sigma_{1}(d)=1+4 \cdot(1-\gamma(d))^{2}+\frac{1}{2} \cdot \sum_{k=1}^{K} \mathcal{N}_{k}
$$

and 


$$
\sigma_{2}(d)=1+2 \cdot \sqrt{1-\gamma(d)}+\frac{1}{2} \cdot \sum_{k=1}^{K} \mathcal{N}_{k}
$$

Both models take into account a standard measurement noise (first addend) and the uncertainty introduced by unprecise attenuation parameters (third addend). The models differ in their second addend which estimates the contribution of the sender-receiver displacement to the error caused by an unprecise pathloss exponent. The effect of creating radio maps for Markov localization on the basis of these functions is described in Sect. 5, where the complete model is also parameterized according to our testbed.

\section{Implementation Details}

Some issues concerning Markov localization on the basis of RSS measurements have so far not been discussed. This section will address the open questions stated in Sect. 2 and provides some additional implementation details.

\subsection{Sensor Model Details}

In Sect. 2 it was left open how $P\left(s_{T} \mid l\right)$ (Eq. 5) should be calculated. Taking the probabilistic propagation model presented in Sect. 3 it is now feasible to determine the likelihood of an RSS reading at a given location. If the expected signal strength calculated for a position and its corresponding uncertainty are interpreted as mean $\mu$ and standard deviation $\sigma$ of a Gaussian distribution then

$$
P\left(a \leq S S_{M} \leq b\right)=\frac{1}{\sqrt{2 \pi} \cdot \sigma} \cdot \int_{a}^{b} e^{-\frac{1}{2}\left(\frac{S S_{M}-S S_{C}}{\sigma}\right)^{2}} d S S_{M}
$$

provides an estimate for the probability $P\left(s_{T} \mid l\right)$ where $S S_{C}$ denotes the calculated RSS and $S S_{M}$ the measured signal strength. The integration boundaries have been set to $a=S S_{M}-\frac{1}{2}$ and $b=S S_{M}+\frac{1}{2}$.

This probability is calculated for each cell in the probability map associated with a base station for which RSS measurements are available. The resulting maps of all base stations - informally put, each representing a guess concerning the MT's position based on the resp. information-subset - are then superimposed in a multiplicative manner.

\subsection{Movement Model Details}

The answer to the question of how to calculate $P\left(l \mid a_{T-1}, L_{T-1}=l^{\prime}\right)$ (Eq. 8), i.e. the probability of reaching one location from another given a movement, has so far been deferred. Before the modelling of movement is tackled it must first be described how movement can be detected in a WLAN without dedicated sensors. At first this presented a major obstacle to the adaptation of Markov localization to WLANs. However it was discovered that the population variance 

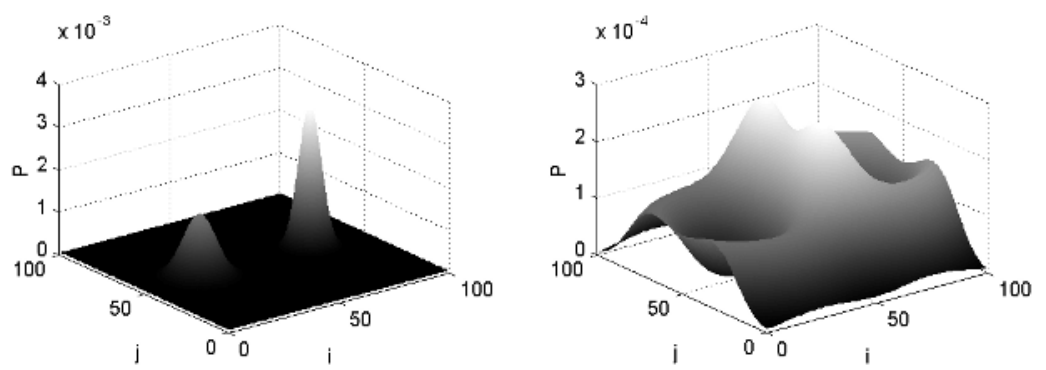

Fig. 2. Belief about MT's position before and after movement model has been applied.

of a sliding mean window, originally used to filter the highly fluctuating raw RSS data, could be used to detect absolute movement quite reliably. Without moving the mobile terminal the variance (of the sliding mean with a window size of 10) seldom exceeded one, even in highly dynamic office environments. When actually moving the mobile terminal the population variance increased to values of six or more at normal walking speed.

The movement model makes use of this phenomenon in order to decide whether the mobile terminal is being moved or not. A population variance above two is interpreted as movement, whereas lower values indicate the MT is stationary. Assuming the mobile terminal has an average speed of $v_{\max }=1.8 \frac{\mathrm{m}}{\mathrm{s}}$ when in movement, then the movement model can estimate the travelled distance using $\Delta s_{M T}=v_{\max } \cdot \Delta t$, where $\Delta t$ denotes the time since the last invocation of the movement model. This is just an approximation of the travelled distance, as the movement detector unfortunately does not indicate direction. This implies that the action $a_{T}$ introduced in Sect. 2 does not describe the directed movement between $t=T-1$ and $t=T$, from position $l \in L$ to position $l^{\prime} \in L$. Rather the implemented action model defines $a_{T}$ as movement from one position $l \in L$ to all positions $l^{\prime} \in L$ within a distance of $s_{M T}$.

As the movement sensor is not accurate concerning the travelled distance of the MT, the underlying uncertainty is modelled as two-dimensional Gaussian distribution, with mean $\mu=\Delta s_{M T}$ and $\sigma=1$. The effect of applying the movement model is clarified in Fig. 2 The left illustration shows the belief about the MT's position at time $t=T-1$. The probability map has two peaks, indicating that there are two positions the MT is likely to be located at. To reduce computational complexity the action model is only applied to probabilities above a certain threshold. In this example the action model is only applied to the maximum of each peak. The right illustration shows the belief at time $t=T$ after movement has been detected. The action model is applied to the two peak values and the resulting probability distributions are additively superimposed. The superimposition of the two volcano cones is apparent - note how the cones are weighted by the probability of their origin.

The outlined scheme provides a means to calculate the desired $P\left(l \mid a_{T-1}, L_{T-1}=l^{\prime}\right)$; tests have proven the estimation of the distance travelled by the MT's between two invocations to be sufficiently accurate. 
Table 1. Object classes and their parameters.

\begin{tabular}{|c|l|r|r|}
\hline object class $k$ & description & attenuation $\eta_{k}^{\text {Wall }}$ & $c_{k}$ \\
\hline \hline 1 & window & $6.0 \mathrm{dBm}$ & 2 \\
\hline 2 & thick concrete & $15.0 \mathrm{dBm}$ & 7 \\
\hline 3 & doors & $2.0 \mathrm{dBm}$ & 3 \\
\hline 4 & light wall & $4.0 \mathrm{dBm}$ & 7 \\
\hline 5 & steel locker & $7.0 \mathrm{dBm}$ & 1 \\
\hline 6 & thin concrete & $5.0 \mathrm{dBm}$ & 1 \\
\hline
\end{tabular}

\subsection{Probability Map Initialization}

Before the first iteration of the algorithm the probability map $\operatorname{Bel}\left(L_{t=0}\right)$ needs to be initialized to reflect the system's knowledge (or at least belief) about the MT's location. In principle two cases need to be considered. In the first case information about the MT's location is available and can be used to initialize the map. This information can be provided by external intervention (e.g. querying the user about his location) or by means of a user profile. In the second case no information pertaining to the MT's initial location is available, which is modelled by initializing $\operatorname{Bel}\left(L_{t=0}\right)$ equally distributed, i.e. all $l \in L$ are equally probable.

The current implementation has no notion of users but only locates terminals. This is done mainly for reasons of privacy, as the WhereMops-system is being used as a research and production platform. Hence the system cannot work on the basis of user profiles and initializes the probability map equally distributed.

\section{Experimental Evaluation}

This section presents the results of the experimental evaluation and compares them with the results obtained of related systems. The investigated properties include the absolute positioning errors both in case of line-of-sight (LOS) and without line-of-sight (NLOS) with no movement, the influence of the uncertainty factor in the radio maps and the accuracy of the estimated positioning error.

All experiments were conducted in the office wing of the Chair for Communication and Distributed Systems at Aachen University depicted in Fig. 1. The wing has a dimension of about $40 \mathrm{~m} \times 15 \mathrm{~m}$ and is covered by three access points placed on this floor, which are marked with circles in Fig. 1 The radio map was generated using the parameters listed in Table 1. The chosen cell size for all maps was $0.5 \mathrm{~m} \times 0.5 \mathrm{~m}$. Furthermore $n_{P}\left(d_{0}\right)$ was set to $-37 \mathrm{dBm}$ with $d_{0}=2 \mathrm{~m}$ and $\eta_{F S}=2$.

The following describes the conducted experiments and their results. The positioning error is defined as the Euclidian distance between the true position and the absolute maximum of the probability density function describing the belief about the MT's state. 


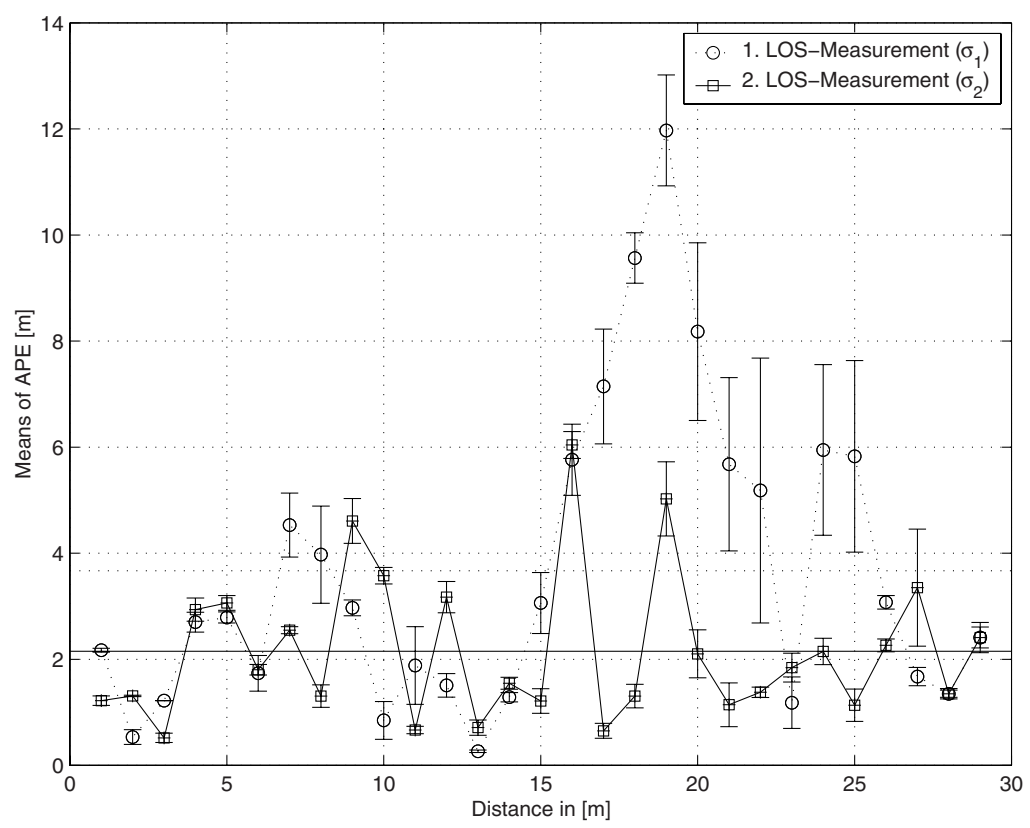

Fig. 3. Absolute positioning error under LOS conditions.

\subsection{Results of LOS-Measurements}

The LOS-measurements were conducted twice to examine the effect of the different uncertainty models (Eq. 111) and (Eq. 12) on the absolute positioning error. Though this series is categorized as being under LOS-conditions it must be noted that all times there is only line-of-sight to one access point.

The measurements were taken at locations in the hallway spaced apart by one meter and marked by a cross in Fig. 1, In both series of measurements the terminal was located periodically every $5 \mathrm{sec}$. After 10 iterations the current probability density, i.e. the belief about the terminal location, was reset by an equal distribution, to capture the convergence of the belief, i.e. probability distribution. In the first series this is done five times resulting in 50 location calculations per measurement point, while all other measurements repeated this procedure 10 times yielding 100 calculations per measurement point.

Figure 3 shows the mean of the 50 resp. 100 location calculations per measurement point. The error bars give the $95 \%$ confidence interval. The distance is given in relation to the LOS access point on the left side of the hallway outside the seminar room abbreviated by "SR".

The first series of measurements started very promising with distance errors in the range of $1 \mathrm{~m}-4 \mathrm{~m}$, but then exposed great errors of up to $12 \mathrm{~m}$ in an area about $16 \mathrm{~m}-22 \mathrm{~m}$ from the measurement's origin. The estimated locations strongly tended towards the left-most access point, which indicates that the measured RSS is higher than the calculated RSS. Indeed the RSS readings of 
the LOS access point were about 5-10 dBm higher than predicted by the model. As all other values in the radio map corresponded well with measured values, it is assumed that the deviation is caused by constructive multi-path propagation.

The experience gained from this first series of measurements motivated the change of the uncertainty model in the radio map module as described in Sect. 3.2. As the RSS measurements deviated more strongly from the empirical propagation model than estimated by $\sigma_{1}$, the uncertainty model $\sigma_{2}$ was defined to assign higher uncertainties even to small transmitter-receiver separations. In the second series of LOS-measurements (using $\sigma_{2}$ ) the resulting error distance dropped significantly, especially in the previously problematic area. Though the effect of an incorrect estimation of the RSS is still visible, all remaining measurements were conducted using the new radio maps created with $\sigma_{2}(d)$.

\subsection{Results of NLOS-Measurements}

The case of NLOS is generally more challenging as the radio signals are subject to more transmission phenomena than in the case of LOS. Furthermore there is a higher chance of errors introduced by false parametrization of the radio propagation model. Despite these adverse conditions the results are quite satisfactory.

One series of measurement per room was carried out, where possible with the terminal placed in the middle of the room. At each location 100 measurements were conducted with a re-initialization of the probability map after 10 iterations (see above).

The cumulative histogram of the absolute positioning errors for the case of LOS and NLOS is shown in Fig. 4, The second series of LOS-measurements using the improved radio propagation model has a median error of $1.5 \mathrm{~m}$ and a $95^{\text {th }}$ percentile of $6 \mathrm{~m}$. The median error in the NLOS-case is $2 \mathrm{~m}$ whereas the $95^{\text {th }}$-percentile amounts to $8.5 \mathrm{~m}$.

\subsection{Estimated Positioning Error}

A novel feature of the proposed indoor geolocation system is the ability to provide an estimate for the distance error of the calculated position. As stated above the maximum of the probability distribution was used as an estimate for the MT's location during system evaluation. To provide an estimate for the distance error, the probabilities of the grid cells surrounding the maximum were added up until a predefined probability threshold was reached. The result is a quasi-circular location area. The radius of this location area is interpreted as the estimated positioning error (EPE) with a confidence level corresponding to the threshold.

During the measurements the EPE at a $90 \%$ confidence level was recorded and afterwards compared to the absolute (i.e. true) positioning error (APE). If the EPE is lower than the APE, then the true position is outside the given location area. If the EPE is greater than the APE then the true position lies within the range stated by the algorithm. For the LOS-measurements $73 \%$ of the EPEs were greater than the corresponding APEs. This is quite satisfactory 


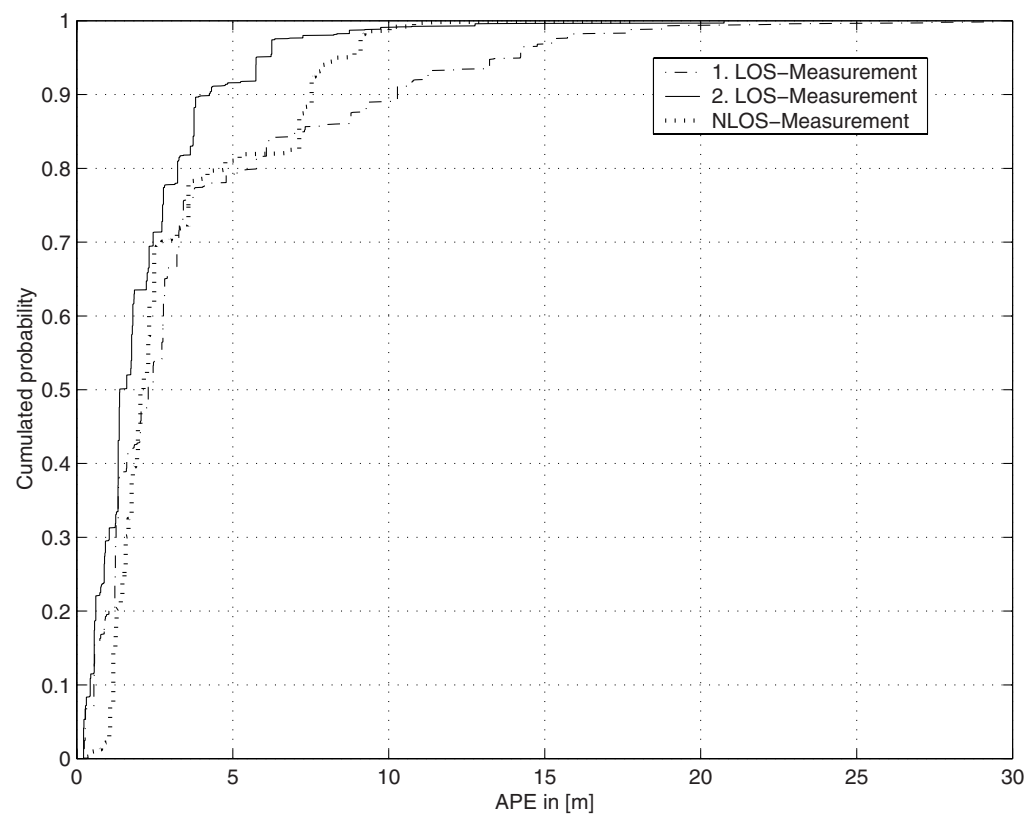

Fig. 4. Cumulative histograms of positioning errors under conditions of LOS and NLOS.

however it is below the desired confidence level of $90 \%$. Surprisingly the NLOSmeasurements showed better results in this respect. Here $89 \%$ of the EPEs were greater than the APEs.

\subsection{Comparison}

Table 2 compares the obtained results with those of related location determination systems presented by Bahl and Padmanabhan in 2 and by Youssef et al. in [4. The location determination system proposed in this paper performs considerably better than the RADAR-system even in the case of NLOS. In contrast the schemes presented in [4] apparently outperform the Markov localization scheme.

Table 2. Comparison of positioning errors.

\begin{tabular}{|r||r|r|r|r|}
\hline System & $25 \%$ & $50 \%$ & $75 \%$ & $95 \%$ \\
\hline \hline Markov-Localization LOS & $0.6 \mathrm{~m}$ & $1.5 \mathrm{~m}$ & $2.8 \mathrm{~m}$ & $6.0 \mathrm{~m}$ \\
\hline Markov-Localization NLOS & $1.5 \mathrm{~m}$ & $2.0 \mathrm{~m}$ & $3.6 \mathrm{~m}$ & $8.5 \mathrm{~m}$ \\
\hline RADAR-System & $1.9 \mathrm{~m}$ & $2.9 \mathrm{~m}$ & $4.7 \mathrm{~m}$ & - \\
\hline Joint Clustering & $0.6 \mathrm{~m}$ & $1.1 \mathrm{~m}$ & $1.2 \mathrm{~m}$ & $2.1 \mathrm{~m}$ \\
\hline Incremental Triangulation & $0.8 \mathrm{~m}$ & $1.2 \mathrm{~m}$ & $1.5 \mathrm{~m}$ & $3.4 \mathrm{~m}$ \\
\hline
\end{tabular}


However it is very likely that this is due to the use of reference measurements for creating a radio map. It is questionable whether the high effort for creating radio maps by measurements justifies the achieved accuracy. Aachen University for example is spread over 150 buildings with a total usable floor space of around $300.000 \mathrm{~m}^{2}$ - taking $300 \mathrm{sec}$ reference measurements for every $2 \mathrm{~m}^{2}$ as suggested in [4] would require at least 4.5 person-years assuming an eight-hour working day.

Furthermore the search-space of the implemented system is not as restricted as those in 2] and 4]. At all times every grid square presented a potential location of the mobile terminal. In contrast the works presented in [2] and 44] naturally restrict their search-space to those locations that were part of their offline measurements, which in both cases only covered hallways.

\section{Conclusions and Future Work}

Determining the position of mobile terminals within buildings on the basis of WLAN signal strength is extremely difficult, due to dynamic environments and complex radio propagation mechanisms. This paper presented the design and implementation of a probabilistic location determination algorithm based on the concept of Markov localization. To avoid time-expensive reference measurements a probabilistic radio propagation model was developed, which provides an estimate for the inaccuracies of the model.

The experimental results are very satisfactory especially considering the use of a radio propagation model instead of reference measurements to provide a data basis. The conducted experiments show that the proposed technique can provide a median error of less than $2 \mathrm{~m}$ even when there is no line-of-sight to an access point. The $90^{t h}$-percentile is around $4 \mathrm{~m}$ under line-of-sight conditions.

In order to investigate the effects of the many parameters of the Markov localization algorithm more efficiently a simulator is currently being developed. The simulator will chose a position on a map and generate RSS values according to previously recorded histograms of real measurements. This simulator can also be used to evaluate the position estimation of a moving terminal, which can hardly be done in practice.

Finally the interpretation of the probability density representing the belief about the mobile terminal's position needs to be analyzed. The maximum of the density function is in general a good candidate for the location estimate. However finding the smallest location area with a given error probability could improve the accuracy.

\section{References}

1. IEEE 802.11 WG, Wireless LAN Medium Access Control (MAC) and Physical Layer (PHY) Specifications, 8802-11: 1999 (ISOIEC) (IEEE Std 802.11, 1999 Edition) Information technology - Telecommunications and information excahnge between systems - Local and metropolitan area networks - Specific Requirements Part 11, 1999. 
2. P. Bahl, V.N. Padmanabhan, RADAR: An In-Building RF-based User Location and Tracking System, Proceedings of IEEE Infocom 2000, Tel-Aviv, Israel.

3. A. Kishan, M. Michael, S. Rihan, R. Biswas, Halibut: An Infrastructure for Wirelss LAN Location-Based Services, Technical Report, Stanford University, June 2001.

4. M.A. Youssef, A. Agrawal, A.A. Shanka, S.H. Noh, A Probabilistic ClusteringBased Indoor Location Determination System, Technical Report, CS-TR-4340, March, 2002.

5. P. Castro, P. Chiu, T. Kremenek, R. Muntz, A Probabilistic Location Service for Wireless Network Environments, Ubiquitous Computing 2001, Atlanta, GA, September 2001.

6. D. Fox, W. Burgard, S. Thrun, Markov Localization for Mobile Robots in Dynamic Environments, Journal of Artificial Intelligence Research 11, Pages 391-427, 1999.

7. M. Hassan-Ali, Using Ray-Tracing Techniques in Site-Specific Statistical Modeling of Indoor Radio Channels, Ph. D. Dissertation, Worcester Polytechnic Institute, Worcester, MA, 1998.

8. P. Harley, Short Distance Attenuation Measurements at 900MHz and 1.8GHz Using Low Antenna Heights for Microcells, IEEE JSAC, Vol. 7, No. 1, January 1989.

9. S.Y. Seidel, T.S. Rappaport, $914 \mathrm{MHz}$ Path Loss Prediction Models for Indoor Wireless Communications in Multifloored Buildings, IEEE Transactions on Antennas and Propagation, Vol. 40, No. 2, February 1992.

10. K.W. Cheung, J.H.M. Sau, R.D. Murch, A New Empirical Model for Indoor Propagation Prediction, IEEE Transactions on Vehicular Technologies, September 1997.

11. M. Wallbaum, WhereMoPS: An Indoor Geolocation System, The 13th IEEE International Symposium on Personal, Indoor and Mobile Radio Communications, Lisboa, Portugal, September 2002. 